Psychother Psychosom 2012;81:380-382

DOI: $10.1159 / 000337176$

\section{Childhood Abuse, Sexual Function and Cortisol Levels in Eating Disorders}

\author{
Giovanni Castellinia , Lorenzo Lelli ${ }^{a}$, Carolina Lo Sauro a, \\ Linda Vignozzib ${ }^{\mathrm{b}}$, Mario Maggi ${ }^{\mathrm{b}}$, Carlo Faravellic, Valdo Ricca ${ }^{\mathrm{a}}$ \\ aPsychiatric Unit, Department of Neuropsychiatric Sciences \\ and ${ }^{\mathrm{b}}$ Unit of Endocrinology, Department of Clinical \\ Physiopathology, Florence University School of Medicine, \\ and ${ }^{\mathrm{C}}$ Department of Psychology, University of Florence, \\ Florence, Italy
}

Several studies assessed the possible associations between eating disorders (EDs) and sexual functioning [1]. Sexual dysfunctions are common in EDs [2]; however, only few studies have attempted to explain the mechanisms underlying them [3]. Childhood abuse (CA) has been considered as a possible moderator of the sexual dysfunction in EDs [4], considering that a history of $\mathrm{CA}$ is a well-documented risk factor for development and maintenance of EDs [1], and it is often associated with sexual dysfunction in adulthood [5]. Hypothalamus-pituitary-adrenal (HPA) axis hyperactivity is well documented in anorexia nervosa (AN) and bulimia nervosa $(\mathrm{BN})$ during the acute phase of the illness [6]. Furthermore, stress response to sexual stimuli in subjects with CA has been reported [5]. Considering the above lines of evidence, we hypothesized a different relationship between sexual functioning and cortisol response according to the presence/absence of a history of CA.

The present study investigated the role of CA as a moderator of the relationship between sexual function and different biological markers in a sample of $78 \mathrm{ED}$ female patients $(41 \mathrm{BN}$, and 37 AN patients), and in 68 age- and gender-matched healthy control (HC) subjects. Patients attending the Outpatient Clinic for Eating Disorders between January 2010 and August 2011 were enrolled in the study, provided they met the following inclusion criteria: female gender, age between 18 and 60 years, a current diagnosis of AN or BN according to DSM-IV criteria (Diagnostic and Statistical Manual of Mental Disorders) evaluated by means of a face-to-face interview (Structured Clinical Interview for DSM-IV). The exclusion criteria were as follows: previous surgical treatment which may interfere with sexual activity (e.g. hysterectomy, episiotomy), current use of antipsychotic medications, absence of sexual activity during the previous 30 days, illiteracy, mental retardation, comorbid schizophrenia, bipolar I disorder. Twenty-two ED patients and $3 \mathrm{HC}$ were taking benzodiazepines and/or antidepressants. The following self-reported questionnaires were administered: Eating Disorder Examination Ques- tionnaire, a 38-item questionnaire, assessing the core psychopathological features of EDs, Barratt Impulsiveness Scale [7], composed by 30 questions concerning control of thoughts and behavior; the Female Sexual Function Index (FSFI), a 19-item self-report instrument that consists of 6 subscales, each of them relating to a specific sexual domain (desire, arousal, lubrication, orgasm, satisfaction, pain) [8]. The Childhood Experience of Care and Abuse Questionnaire was administered in order to evaluate physical and sexual abuse, maternal and paternal antipathy and neglect; it assesses the degree of severity, the frequency and relationship to perpetrator/caregiver of adversity or abuse [9]. Finally, Beck Depression Inventory, and State-Trait Anxiety Inventory were administered. Blood samples were drawn in the morning ( 8 a.m.) after an overnight fast for determination of cortisol levels. All the patients were asked to provide their written informed consent. The study protocol was approved by the Ethics Committee of the Institution. For between-group comparisons (HC vs. EDs; AN vs. BN) $\chi^{2}$, Mann-Whitney U test, and univariate analysis of variance were applied. Correlation analyses (Spearman test), and subsequently linear regression analyses were performed in the whole sample, and within each group, to assess the associations of FSFI scores with clinical variables and biological markers. Finally, general linear model (GLM) was used to examine the moderating effect of diagnosis (HC vs. EDs) and abuse (no history of CA vs. history of CA) on significant interactions obtained in the univariate analyses.

AN patients showed lower BMI (16.8 $\pm 2.5 ; \mathrm{F}=61.9 ; \mathrm{p}<$ $0.001)$ compared with $\mathrm{BN}(22.5 \pm 2.5)$ and $\mathrm{HC}(21.5 \pm 2.2)$. Compared with HC subjects, ED patients showed lower frequency of sexual intercourse, and lower scores in all the FSFI domains (arousal, lubrication, orgasm, satisfaction, pain, and FSFI total score; all $\mathrm{p}<0.01$ ), with the exception of desire domain. No significant differences were found between $\mathrm{AN}$ and $\mathrm{BN}$, in terms of sexual functioning (sexual intercourses and FSFI scores), and psychopathology (ED symptoms, depression and anxiety). A history of CA was found to be more frequent in ED patients compared with $\mathrm{HC}$, when considering both physical abuse (30.1 vs. $8.8 \% ; \chi^{2}=10.0 ; \mathrm{p}=0.002$; mean age of abuse $\pm \mathrm{SD}=7.6 \pm 3.1$ years), and sexual abuse ( 12.3 vs. $2.9 \% ; \chi^{2}=4.3 ; \mathrm{p}=0.038$; mean age of abuse $\pm \mathrm{SD}=9.8 \pm 4.0$ years). Considering the perpetrator, father was implied in $72 \%$ of physical abuse cases, and $60 \%$ of sexual abuse cases; mother was implied in $16 \%$ and $10 \%$ of physical and sexual abuse cases, respectively. ED patients reporting sexual abuse showed higher objective $(z=2.6 ; p=0.008)$ and subjective $(\mathrm{z}=2.5 ; \mathrm{p}=0.010)$ binge eating frequency, and Barratt Impulsiveness Scale scores $(\mathrm{z}=2.5 ; \mathrm{p}=0.012)$, compared with the rest of ED patients. Depression was inversely associated with FSFI scores in HC, but no significant effects of general psychopathology and pharmacological treatments on sexual functioning and cortisol levels were detected in ED patients. Other com-

\section{KARGER}

Fax +4161306 1234 E-Mail karger@karger.ch www.karger.com
(C) 2012 S. Karger AG, Basel

$0033-3190 / 12 / 0861-0380 \$ 38.00 / 0$

Accessible online at: www.karger.com/pps
Valdo Ricca, MD

Psychiatric Unit, Department of Neuropsychiatric Sciences Florence University School of Medicine, Largo Brambilla 3 IT-50134 Firenze (Italy)

Tel. +39055 794 7487, E-Mail valdo.ricca@unifi.it 
GLM (dependent variable: cortisol level at 8 a.m.)

\begin{tabular}{lcrl}
\hline & $\beta$ & $\mathrm{t}$ & $\mathrm{p}$ \\
\hline Age & -0.01 & -1.25 & 0.21 \\
Desire & 0.68 & 3.25 & 0.002 \\
Diagnosis & 0.31 & 1.68 & 0.09 \\
Desire $\times$ diagnosis interaction & 0.002 & 0.01 & 0.99 \\
Abuse & 0.29 & 1.30 & 0.19 \\
Desire $\times$ abuse interaction & 0.54 & 2.11 & 0.03 \\
\hline
\end{tabular}

Not abused

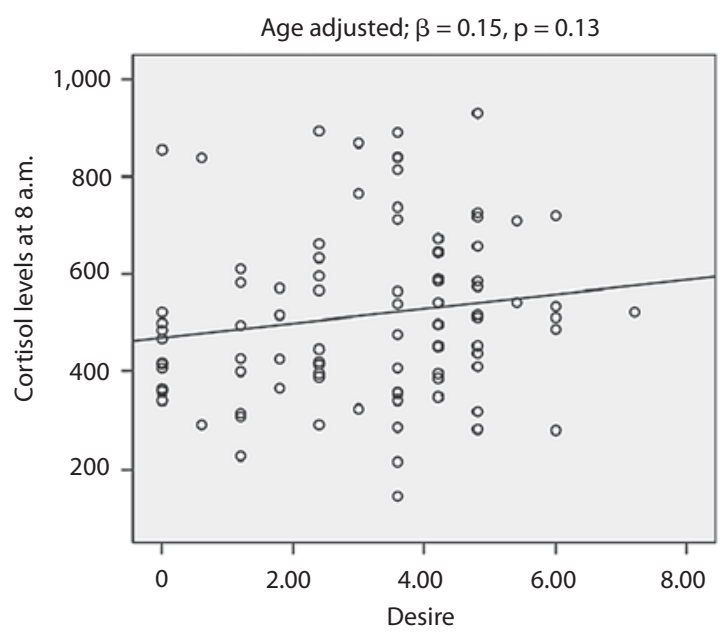

Abused

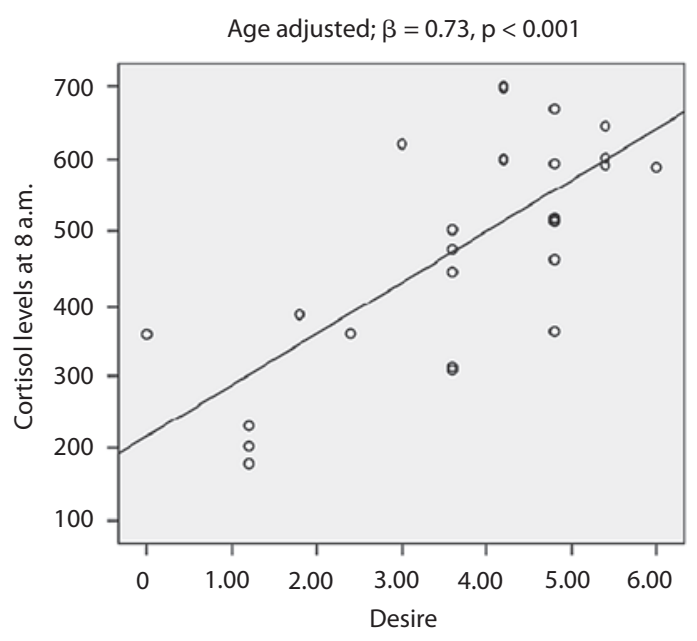

Fig. 1. Moderators of the relationship between sexual desire and cortisol levels. Desire was a subscale of the FSFI. Diagnosis and abuse were coded as dummy variables: $0=\mathrm{HC}$ subjects, $1=\mathrm{ED}$ patients; 0 = subjects without a history of CA (physical and sexual), 1 = subjects with a history of sexual abuse. GLM = General Linear Model. Graphs report results from linear regression analyses. parisons or correlations were not significant. As far as biological markers are concerned, all the FSFI scores showed a positive association with cortisol levels. The higher significant effect was detected for the desire domain $(\beta=0.23 ; \mathrm{p}=0.01)$. GLM analysis (age adjusted) showed a significant effect of desire by diagnosis on cortisol levels ( $\beta=0.48 ; p=0.042)$. Therefore, when the interaction was broken down, the increased desire was associated with increased cortisol levels in the ED group $(\beta=0.36, p=0.005)$, but not in the HC group. A significant effect of desire by CA (physical and sexual) was found (fig. 1). When entering the interaction in the previous model, the interaction with diagnosis lost its significance. Furthermore, when the interaction was broken down, the increased desire was associated with increased cortisol levels in the CA group, but not in the non-CA group (fig. 1). A significant effect of desire by age at abuse was found $(\beta=0.08, p<0.01)$, with lower age associated with higher effect of desire on cortisol levels.

Our findings suggest a complex relationship between history of CA, ED psychopathology, and sexual and hypothalamus-pituitary-adrenal functioning. Both physical abuse and sexual abuse represented a significant risk factor for development of ED psychopathology [1], and they were associated with more frequent impulsive behaviors. In our study, CA did not show a direct effect on sexual functioning or on cortisol levels. The positive association between FSFI scores and cortisol levels seems to be in contrast with the observation that in healthy women physiological sexual arousal is associated with a slight decrease in cortisol levels [10]. However, a possible explanation for this result was provided by the moderator models we performed, showing that the interaction of sexual desire with CA was significant, and, accordingly, the effect of sexual desire on cortisol levels was observed only in the CA group. These data confirmed an exploratory analysis by Rellini et al. [5], reporting greater cortisol responses associated with greater perceived states of physiological sexual arousal in subjects reporting CA, while for the non-CA subjects, greater cortisol response was associated with lower perceived physiological sexual responses [5]. It can be hypothesized that in people who have been abused during childhood, the emotional involvement in the sexual relationship would result in activation of the mechanisms of stress. Given that the traumatic experiences of CA frequently involved sexual stimuli, it is possible that when perceiving sexual desire, these women would experience an automatic fear response which in turn determines a significant increase in cortisol levels. Larger, prospective studies are needed in order to confirm these preliminary data.

\section{Disclosure Statement}

The authors declare no conflict of interest.

\section{References}

1 Jacobi C, Hayward C, de Zwaan M, Kraemer HC, Agras WS: Coming to terms with risk factors for eating disorders: application of risk terminology and suggestions for a general taxonomy. Psychol Bull 2004; 130:19-65.

2 Morgan JF, Lacey JH, Reid F: Anorexia nervosa: changes in sexuality during weight restoration. Psychosom Med 1999;61:541-545.

3 Pinheiro AP, Raney TJ, Thornton LM, Fichter MM, Berrettini WH, Goldman D, Halmi KA, Kaplan AS, Strober M, Treasure J, Woodside DB, Kaye WH, Bulik CM: Sexual functioning in women with eating disorders. Int J Eat Disord 2010;43:123-129. 
4 Mangweth-Matzek B, Rupp CI, Hausmann A, Kemmler G, Biebl W: Menarche, puberty, and first sexual activities in eating-disordered patients as compared with a psychiatric and a non-psychiatric control group. Int J Eat Disord 2007;40:705-710.

5 Rellini AH, Hamilton LD, Delville Y, Meston CM: The cortisol response during physiological sexual arousal in adult women with a history of childhood sexual abuse. J Trauma Stress 2009;22:557-565.

-6 Lo Sauro C, Ravaldi C, Cabras PL, Faravelli C, Ricca V: Stress, hypothalamic-pituitary-adrenal axis and eating disorders. Neuropsychobiology 2008;57:95-115.

7 Patton JH, Stanford MS, Barratt ES: Factor structure of the Barratt Impulsiveness Scale. J Clin Psychol 1995;51:768-774.
8 Rosen R, Brown C, Heiman J, Leiblum S, Meston C, Shabsigh R, Ferguson D, D’Agostino R Jr: The Female Sexual Function Index (FSFI): a multidimensional self-report instrument for the assessment of female sexual function. J Sex Marital Ther 2000;26:191-208.

$\checkmark 9$ Bifulco A, Bernazzani O, Moran PM, Jacobs C: Childhood Experience of Care and Abuse Questionnaire (CECA.Q): validation in a community series. Br J Clin Psychol 2005;44:563-581.

10 Exton NG, Truong TC, Exton MS: Neuroendocrine response to filminduced sexual arousal in men and women. Psychoneuroendocrinology 2000;25:187-199. 